\title{
Laboreal
}

Volume 13 NN$^{\circ} 2$ | 2017

Varia

\section{Fiabilización de las decisiones en la sala de control de una central nuclear: el papel de la argumentación en la resolución colectiva de problemas}

Fiabilização das decisões na sala de comando de uma central nuclear: o papel da argumentação na resolução coletiva de problemas

Fiabilisation des décisions de conduite en salle de commande d'une centrale nucléaire : le rôle de l'argumentation dans la résolution collective de problèmes Reliability in the decisions taken in the control room of a nuclear plant: the role of argumentation in the collective problems resolution

\section{Johanna Mérand}

\section{OpenEdition}

\section{Journals}

\section{Edición electrónica}

URL: http://journals.openedition.org/laboreal/340

DOI: $10.4000 /$ laboreal.340

ISSN: 1646-5237

Editor

Universidade do Porto

\section{Referencia electrónica}

Johanna Mérand, « Fiabilización de las decisiones en la sala de control de una central nuclear: el papel de la argumentación en la resolución colectiva de problemas », Laboreal [En línea], Volume 13 №2 I 2017, Publicado el 01 diciembre 2017, consultado el 25 septiembre 2020. URL : http://

journals.openedition.org/laboreal/340; DOI : https://doi.org/10.4000/laboreal.340

Este documento fue generado automáticamente el 25 septiembre 2020.

\section{(c) (1) \&}

Laboreal está licenciado com uma Licença Creative Commons - Atribuição-NãoComercial 4.0 Internacional. 


\section{Fiabilización de las decisiones en la sala de control de una central nuclear: el papel de la argumentación en la resolución colectiva de problemas}

Fiabilização das decisões na sala de comando de uma central nuclear: o papel da argumentação na resolução coletiva de problemas

Fiabilisation des décisions de conduite en salle de commande d'une centrale nucléaire : le rôle de l'argumentation dans la résolution collective de problèmes Reliability in the decisions taken in the control room of a nuclear plant: the role of argumentation in the collective problems resolution

Johanna Mérand

\section{REFERENCIA}

Mérand, J. (2016). Fiabilisation des décisions de conduite en salle de commande d'une centrale nucléaire : le rôle de l'argumentation dans la résolution collective de problèmes. Thèse de doctorat en Ergonomie. Université Paris-Sud XI, Orsay.

\section{NOTA DEL EDITOR}

http://dx.doi.org/10.15667/laborealxiii0217jmes

Manuscrito recibido en: Agosto/2016

Aceptado tras peritaje: Noviembre/2017 
Jurado de tesis: Pierre Falzon (Presidente); Françoise Darses (Director da tesis); Cecilia de la Garza (Co-Directora de tesis); Nicolas Ambrosi, Christine Chauvin e Jean-François Vautier (Jurados).

\section{Contexto industrial}

1 La tesis tiene como objetivo la resolución colectiva de problemas en el control de situaciones accidentales de un sistema dinámico y complejode alto riesgo La tesis se enmarca dentro de un proyecto de diseño de una sala de control de un nuevo reactor nuclear, dirigido por equipos multidisciplinarios de EDF. Este programa pretende evaluar la acoplamiento entre el factor humano, los sistemas de control, las interfaces humano-máquina y la organización de equipo, antes de la puesta en marcha del reactor (De La Garza, Labarthe, \& Graglia, 2012; Labarthe \& De La Garza, 2011). Se examinaron dos organizaciones del equipo de control en dos campañas de evaluación sucesivas: una organización compuesta por cinco operadores (orga-5), similar a las existentes en las plantas de producción actuales francesas, y una nueva organización compuesta por cuatro operadores (orga-4). La tesis hace especial hincapié en el impacto de estas modalidades de organización en los procesos colectivos de resolución de problemas, es decir, cuando los operadores construyen una nueva representación del estado de la instalación y/o ajustan el procedimiento de control a la situación.

\section{Marco teórico}

2 Los entornos dinámicos de alto riesgo, como las centrales nucleares, imponen una serie de condiciones particulares en la actividad cognitiva de resolución de problemas. Estas especificidades se han descrito en numerosas ocasiones en la literatura y se integran en modelos más amplios de control y de supervisión de un proceso dinámico (Hoc \& Amalberti, 1995; Klein \& Klinger, 1991; Rasmussen, 1986; Vicente, Mumaw, \& Roth, 2004).

3 En estos de entornos alto riesgo, la complejidad del sistema técnico y la gravedad de las consecuencias potenciales de un error son importantes, especialmente en un contexto de incidentes o accidentes. Sin embargo, en una central nuclear los lapsos de tiempo suelen ser suficientemente amplios para que, ante un problema, el equipo pueda encaminar procesos colectivos profundos de comprensión y tratamiento de dicho problema. Estas interacciones se basan, en gran medida, en la comunicación verbal. En estas situaciones de resolución colectiva de problemas, los operadores recogen, intercambian, cotejan, integran información. En definitiva, cooperan para construir un modelo mental compartido de la situación (Cannon-Bowers, Salas, \& Converse, 1993) en el cual se basan sus decisiones.

El marco teórico de la argumentación, desarrollado por la psicolingüística años atrás, es propicio para el estudio del contenido y de la articulación de las comunicaciones en la resolución colectiva de problemas. Según éste enfoque, argumentar significa convencer al receptor de la pertinencia de su tesis, utilizando aserciones o argumentos razonados en un contexto de incertidumbre (Breton, 2006; Oléron, 1996; Perelman \& OlbrechtsTyteca, 1958/2008). La argumentación posee una doble función (Champaud, 1994; Darses, 2006; Nussbaum, 2008). Es cooperativa ya que al tener un objetivo común 
favorece el desarrollo de interacciones constructivas en las que se crean conjuntamente nuevos sentidos, o nuevas soluciones a partir de las contribuciones individuales (Baker, 1999). También es dialéctica cuando se oponen dos puntos de vista. Refutar, objetar, contradecir, poner en duda, evaluar aportando elementos de apoyo que permiten poner a prueba la validez de las propuestas y los argumentos aportados por los otros miembros del grupo (Rieke, Sillars, \& Peterson, 2005). Esta doble función convierte la argumentación en un proceso potencial de fiabilización de las tomade decisiones, especialmente en la resolución de problemas.

\section{Problemática y organización de la tesis}

5 La tesis sostenida es que los flujos de comunicaciones en el equipo y la actividad argumentativa que desarrollan los operadores en la resolución colectiva de problemas influyen en la calidad de los diagnósticos elaborados y en las toma de decisiones. El sentido de la situación y el plan de acción se construirían y ajustarían conforme a la interacción verbal, aportando argumentos y contraargumentos basados en los conocimientos técnicos, según las condiciones de la situación o las exigencias de organización. Las modalidades de organización, tales como la asignación del personal y la distribución de sus funciones, tendrían un impacto más o menos favorable en el despliegue de procesos argumentativos que tienden a fiabilizar la toma de decisiones colectivas.

6 Estas cuestiones se han formulado e investigado en un proceso dividido en cuatro etapas, cada una de las cuales corresponde a un estudio empírico (véase Figura 1).

7 Las etapas 1 y 2 se centran en la construcción de la problemática y la definición de las opciones conceptuales y metodológicas para el tratamiento de los datos de las etapas siguientes. Estas dos etapas consisten en unos análisis exploratorios de la actividad de los operadores que conforman la nueva organización de equipo (orga-4), la cual no dispone de un equivalente en las plantas francesas existentes de producción. Las describimos brevemente en la sección 5. Las etapas 3 y 4 ilustran la problemática examinando los flujos de comunicación (etapa 3) y la actividad argumentativa (etapa 4) de los equipos en la resolución colectiva de problemas y su impacto en la eficiencia de los equipos. Las describimos de forma más detallada en la sección 6.

8 Estas etapas se basan en escenarios de simulación realistas de operación de una planta nuclear durante fases incidentales/accidentales realizadas en un simulador a escala completa.

Figura 1: Organización de la tesis

\section{População de estudo}

9 Las funciones asumidas por los operadores en los equipos de control orga-4 y orga-5 son relativamente similares.

- El operador encargado de las operaciones de control (op) realiza las acciones de operación. Esta función es realizada por un único operador en los equipos orga-4 y por dos operadores en los equipos orga-5. 
- El supervisor (sup) controla la correcta ejecución de estas acciones, anticipa las acciones de control y se asegura de que la estrategia de operación sea la adecuada en relación con el estado de la planta.

- El jefe de explotación (ce), responsable del equipo, controla la coherencia entre la estrategia de la operacion en curso y el estado de la instalación. Garantiza la interfaz con el puesto de mando de dirección de gestión de crisis.

- El ingeniero de seguridad (is) realiza un control diversificado e independiente del estado de la instalación.

10 Todos los operadores que asumen la función op primero fueron operadores de campo (AT), durante un periodo de tiempo más o menos largo. Los sup han asumido la función op en su carrera profesional. Los ce, de estatuto ejecutivo, en general tienen una formación de ingeniero, como los is.

11 Los operadores no tienen el mismo número de años de experiencia en la operación de una planta nuclear, y esta experiencia puede referirse a procedimientos técnicos diferentes. Los op, sup, ce e is tienen una experiencia en las operaciones de planta que va de 0 a 6 años; de 7 a 14; de 3 a 10 años y de 0 a 5 años respectivamente. Los perfiles de equipo, en términos de experiencia en operación de plantas, varían según el equipo y la organización. Sin embargo, observamos que los tres op de los equipos orga-4 tienen una experiencia en la operación que va de los 3 a los 7 años incluidos mientras que cuatro de los seis op de los equipos orga-5 no tienen ninguna experiencia, aparte de la adquirida durante su formación en un simulador.

El nivel de conocimientos específicos de los operadores sobre el nuevo reactor (nuevos automatismos, especificidades de los sistemas elementales, nuevas estrategias de operación) varía aún más, independientemente de la función asumida y de la organización de equipo. Este nivel depende de la frecuencia de los entrenamientos en simulador, la participación en la validación de los nuevos procedimientos de conducta o interfaces de conducta, etc.

\section{Etapas 1 y 2: construcción de la problemática}

13 La etapa 1 se centró en comprender las tareas y las exigencias cognitivas de los operadores de los equipos orga-4 y del entorno técnico y reglamentario. Se realizaron un estudio de la documentación interna (prescripciones, recomendaciones, estudios internos), observaciones abiertas y entrevistas colectivas exploratorias durante la formación de los equipos en la operación en fases incidentales/accidentales en un simulador a escala completa. Este análisis demostró, entre otros, que los operadores de los equipos orga-4 debían hacer frente a exigencias cognitivas más importantes en las fases de gestión de problemas. Estos problemas solían dar lugar a concertaciones entre los miembros del equipo.

14 La etapa 2 consistió en investigar la gestión colectiva y dinámica de los problemas encontrados por los equipos orga-4, vía el análisis de las interacciones verbales. Hemos definido bajo el término "problema" las situaciones que perturban la aplicación y/o la continuación del procedimiento óptimo de operación del proceso. En total se observaron in situ cinco simulaciones de una duración de unas $2 \mathrm{~h} 30$ cada una con diferentes equipos orga-4. Se analizaron, a posteriori, a través de lasgrabaciones de audio y vídeo. Estos datos se completaron con diez horas de entrevistas colectivas 
postsimulación, dirigidas por los formadores, durante las cuales se tomaron notas sobre las dificultades individuales y colectivas encontradas. El análisis se enfoca en una secuencia de una de las simulaciones observadas que reagrupa numerosos problemas gestionados por el equipo. Cada problema contenido en esta secuencia se ha caracterizado por: 1) su objeto, 2) el número y el contenido de las concertaciones destinadas a gestionar el problema. Una concertación correspondía a una sucesión de intercambios entre uno o varios miembros del equipo, 3) los miembros implicados en cada concertación, por ejemplo, la díada op-sup, la díada sup-ce o la tríada op-sup-ce. Este análisis exploratorio nos permitió mostrar que la gestión colectiva de un problema, a menudo se traduce por una secuencia de varias concertaciones muy cortas (inferiores al minuto). Algunas gestiones de problemas estaban más fraccionadas con el paso del tiempo. Este fraccionamiento parecía estar relacionado en parte con el inicio de procesos cognitivos de más alto nivel para la elaboración de un procedimiento, la construcción de una representación o la realización de un diagnóstico causal del estado de la instalación (comportamientos knowledge- based). Para tratar estos problemas, los operadores del equipo implementan procesos de resolución de problemas. Además, los operadores no parecen estar implicados de la misma forma según su función en el equipo: el op interviene más en la gestión de los problemas con un impacto directo y a corto plazo en la operación de la planta, el ce se centra más en la gestión anticipada de los problemas y en la elaboración de objetivos, el sup aporta información sobre las posibilidades de implementación de estos objetivos.

Después de estas dos etapas, el objeto de la tesis se centró en las situaciones de resolución colectiva de problemas durante las cuales los operadores deben construir una nueva representación del estado de la instalación y/o elaborar un procedimiento de conducta más adaptado a la situación. En estas dos etapas surgieron diferentes cuestiones. Entre otras, el impacto de las modalidades de organización del equipo sobre:

- los flujos de comunicación - ¿Las comunicaciones se distribuyen de forma similar entre los equipos orga-4 y orga- 5 ? $i$ Esta distribución tiene un impacto en la eficiencia del equipo durante la resolución de problemas?

- la participación y el tipo de contribución de los miembros del equipo - ¿La participación y el tipo de contribución de los operadores dependen de la función que asume el operador? ¿De la organización de equipo? ¿Cuál es el impacto en la eficiencia del equipo?

16 A estas modalidades de organización también se añaden factores individuales (experiencia en operación de una planta, conocimientos específicos sobre el proceso) que no se habían tomado en cuenta hasta el momento, pero también susceptibles de influir en la resolución colectiva de problemas y en la eficiencia del equipo. Para investigar estas cuestiones, decidimos recurrir al marco teórico de la argumentación del que se desprende nuestra problemática (véase sección 3).

\section{Etapas 3 y 4: examen de los flujos de comunicación y de la actividad argumentativa en la resolución de problemas}

17 Los flujos de comunicación se estudiaron ampliamente en el pasado (Barth, Schraagen, \& Schmettow, 2015; Park, Jung, \& Yang, 2012). Pero son muy pocos los estudios que han 
examinado la actividad argumentativa en situación dinámica, como en el ámbito medico (Hagler \& Brem, 2008; Lu \& Lajoie, 2008) o en la aeronáutica (Bourgeon, Valot, \& Navarro, 2013). Sin embargo, ninguno propone un modelo de la argumentación en situación dinámica en el cual basarse. El objetivo principal de estas dos etapas es elaborar y probar un modelo de la argumentación que combina los modelos existentes de la toma de decisión en entorno dinámico, en el marco teórico de la argumentación.

\subsection{Método}

\subsubsection{Elaboración de un modelo de la argumentación en entorno dinámico: el modelo ARGUinDSM}

El modelo ARGUinDSM conjuga el marco teórico de la argumentación (Perelman \& Olbrechts-Tyteca, 1958/2008; Toulmin, 1958/2003) con el modelo Dynamic Situation Management (DSM) de Hoc y Amalberti (1995). Este último tiene la particularidad de representar las diferentes etapas del tratamiento de la información como no secuenciales y aportar una arquitectura cognitiva muy detallada.

Figura 2: Modelo de la argumentación en situación dinámica - ARGUinDSM (Mérand \& Darses, 2017)

19 El modelo ARGUinDSM (véase Figura 2) retoma los principales postulados del modelo DSM para trasladarlos a la actividad argumentativa.

20 A cada nivel de abstracción del modelo DSM corresponde una línea argumentativa específica que reagrupa varias funciones argumentativas. Estas se inspiran en los módulos de tratamiento de la información definidos en el modelo DSM, reformulándolos en una perspectiva argumentativa. El modelo presenta tres lí- neas argumentativas, completadas por una cuarta, transversal a las otras tres. Estas líneas se describen a continuación:

- Línea Alimentación de la posición. - Reagrupa las funciones argumentativas para alimentar el proceso de co-construcción del problema y los posicionamientos de cada persona. Encontramos las funciones argumentativas Informar o Solicitar información.

- Línea Construcción de la posición. - Reagrupa las funciones argumentativas para construir una representación compartida del estado-problema y elaborar un plan de acción. Encontramos, por ejemplo, las funciones argumentativas Identificar, Proponer, o incluso Buscar información (con el objetivo de confirmar o apoyar un diagnóstico).

- Línea Crítica de la posición. - Reúne las funciones argumentativas para explicar y evaluar el estado-problema o el plan de acción. Implican razonar a un nivel mayor de abstracción. Encontramos, por ejemplo, las funciones argumentativas Evaluar (positivamente o negativamente) y Explicar.

- Línea Aprobación de la posición. - Reagrupa las funciones argumentativas para validar o invalidar los enunciados emitidos anteriormente por los otros o por uno mismo. Esta línea permite extraer un primer nivel explícito de articulación de los intercambios. Por ejemplo, esta línea engloba las funciones argumentativas Validar, No Validar o Solicitar validación.

Estas líneas contribuyen a establecer una representación colectiva del estado del problema y acciones a implementar para resolverlo. 


\subsubsection{Recoleccción de datos} grabaciones de audio y vídeo ( 36 horas) de la actividad en sala de control. Las anotaciones en tiempo real englobaban seis categorías de hechos observables: 1) las eventos relativos al proceso; 2) las principales acciones de la operación; 3) el avance en las reglas de operacion; 4) las principales comunicaciones en el equipo y con el exterior de la sala de control; 5) las dificultades encontradas en la operación; 6) las posiciones sostenidas por los operadores durante la gestión del problema cuando eran claramente identificables. Estos datos se completaron con 36 horas de entrevistas colectivas postsimulación, dirigidas por el equipo de evaluación del proyecto. Estas entrevistas permitieron profundizar nuestra comprensión de las situaciones problemáticas encontradas por los equipos y recolectar elementos relativos a la representación mental y al posicionamiento de los actores respecto al estado de la situación y acciones previstas y realizadas para resolver los problemas. Seguidamente estos datos se completaron con 15 entrevistas individuales realizadas a posteriori con los operadores de los equipos orga -5 .

\subsection{Análisis de los datos}

Las comunicaciones verbales emitidas durante las simulaciones se transcribieron para identificar eventos-problemas. Un evento- problema reagrupa el conjunto de intercambios para la resolución de un problema particular, por ejemplo, una no adecuación del procedimiento prescrito de operación dada la situación. Un evento-problema podía ser objeto de una o varias concertaciones, sucesivas o no, entre los miembros del equipo, por ejemplo, entre op-sup, sup-ce, op-sup-ce, etc.

25 Cada evento-problema se caracterizó por su objeto, su contexto, la cronología de las díadas y tríadas de actores implicados en la resolución del problema, los diagnósticos planteados y las decisiones tomadas durante y/o después de la evento-problema. A continuación, cada concertación se dividió en propuestas significativas, denominadas unidades de sentido. Para dar cuenta de los flujos de comunicación entre los miembros del equipo (etapa 3), cada unidad de sentido se asoció a su emisor y se contabilizó como perteneciente a una u otra de las díadas o tríadas.

26 Seguidamente, se especificó el contenido de las unidades de sentido mediante un esquema de codificación predicado/argumentos elaborado a partir del modelo ARGUinDSM (etapa 4). A cada unidad de sentido corresponde una función argumentativa que contribuye a alimentar, construir, criticar o aprobar una posición sobre el problema. Esta función argumentativa trata un objeto pasado, presente o futuro. Puede encajar con otra función argumentativa emitida anteriormente, cuando contribuye a validarla o a invalidarla. También se pueden aportar elementos de apoyo para algunas funciones argumentativas. Es el caso, por ejemplo, para Evaluar o Proponer. Finalmente se evaluó la eficiencia de los equipos en función de la exactitud de los diagnósticos elaborados y del carácter adaptado de las decisiones tomadas durante las 
eventos-problema. Dos expertos juzgaron estos diagnósticos y decisiones: un experto en Fiabilidad Humana y un experto en Ergonomía.

\subsection{Principais resultados}

28 As etapas 3 e 4 fornecem resultados complementares quanto à forma como os operadores discutem e articulam os respetivos intercâmbios para a resolução dos evento-problema. Estes resultados dizem principalmente respeito às funções da equipa op, sup e ce. 0 es está, com efeito, menos envolvido na resolução dos eventos-problema em virtude de o seu controlo independente necessitar de uma postura mais recuada em relação aos membros da equipa de operação.

\subsubsection{Uma comunicação mais distribuída nas equipas orga-5}

29 Nas equipas orga-5, a proporção de unidades de sentido emitidas pela função op é maior (op: $\mathrm{c} 2(\mathrm{ddl}=1)=31,2 \mathrm{p}<0,001)$ ao passo que a do sup, maioritária nas equipas orga-4 diminui e tende a juntarse à do $c e($ sup: $\mathrm{c} 2(\mathrm{ddl}=1)=11,6 \mathrm{p}<0,001)$. 0 op dispõe de margens de manobra maiores para participar na resolução coletiva de problemas. A percentagem dos intercâmbios que reúne op-sup-ce é também superior (op-sup-ce: c2 $(\mathrm{ddl} 1)=95,97 ; \mathrm{p}<0,001)$ ao passo que os intercâmbios entre sup-ce diminuem (ce-sup:c2 $(d d l 1)=48,43 ; p<0,001)($ cf. Figura 3$)$.

Gráfico 1: Flujo de comunicación, según la organización de equipo operadores que asumen funciones diferentes en los equipos de 5 operadores.

\subsubsection{Una movilización más importante de la línea Alimenta ción y menos importante de la línea Construcción en los equipos orga-5}

El análisis comparativo de las líneas utilizadas por los equipos con mayor eficiencia con las utilizadas por los otros equipos no muestran una diferencia significativa entre estas dos organizaciones. Parece que no hay correlación directa entre la actividad argumentativa y la eficiencia de los equipos. En cambio, observamos efectos moderados de la organización sobre los procesos argumentativos. Así pues, la línea Construcción se utilizó menos en los equipos orga-5 $(\mathrm{x} 2(\mathrm{df}=1)=8.19 ; \mathrm{p}=0.004)$ mientras que la línea Alimentación se utilizó más $(\mathrm{x} 2(\mathrm{df}=1)=4.29 ; \mathrm{p}=0.038)$ (véase Figura 4$)$.

Figura 4: Movilización de las líneas argumentativas, según la organización de equipo

Un análisis más profundo de las funciones argumentativas movilizadas en la línea Construcción demuestra que los equipos orga- 5 formulan más enunciados para buscar información (SEARCH: $\mathrm{x} 2(\mathrm{df}=1)=19.42 ; \mathrm{p}<0.001)$ y menos enunciados para Identificar el estado de la instalación (IDEN: $\mathrm{x} 2(\mathrm{df}=1)=10.27 ; \mathrm{p}=0.0013)$ y Proponer soluciones a los (PROP: $\mathrm{x} 2(\mathrm{df}=1)=4.89 ; \mathrm{p}=0.027)$.

\subsubsection{Una postura más crítica del OP en los equipos orga-5}

Para examinar la influencia de la organización en la función argumentativa de los equipos, según la función asumida en el equipo, distinguimos "la implicación" de la 
"contribución" de los operadores. La implicación de un operador es definida como su compromiso en las cuatro líneas argumentativas. Su contribución se define como su aportación a cada una de las líneas argumentativas desarrolladas por el equipo. La Figura 5 describe cómo se miden estos dos conceptos.

Figura 5. Medidas de la implicación y de la contribución individuales a las líneas argumentativas comunicación más repartida entre los miembros de los equipos orga-5. Es muy probable que la carga de trabajo de la función op, repartida entre dos miembros en los equipos orga-5, permita a los operadores que realizan esta función: i) estar más disponibles para participar en la resolución de problemas: ii) adoptar una postura más crítica mostrando más distancia respecto a la situación. Si estos efectos no han tenido incidencia en la eficiencia de los equipos en las situaciones observadas, creemos que son deseables y favorables para la fiabilización de las decisiones en la sala de control.

Los equipos orga-5 también parecen demostrar una mayor comprensión de los problemas. Tendrían más libertad para alimentar la resolución de problemas y para construir una representación del problema y del plan de acción con comportamientos de búsqueda de información. Las identificaciones serían más exactas y las proposiciones más adaptadas, y posteriormente no necesitarían una reformulación. El tiempo de construcción de una representación del problema y de un plan de acción disminuiría. Estas interpretaciones están corroboradas por observaciones más cualitativas que reflejan equipos orga-5 más serenos en la sala de control. 
En cambio, no se ha observado ninguna relación entre la actividad argumentativa desarrollada y la eficiencia de los equipos. Este resultado puede explicarse por diferentes factores. Por una parte, el tamaño de la muestra, limitado al alcance del estudio comparativo entre los equipos. Aunque estos seis equipos constituyen una muestra representativa de los futuros equipos de operación de la planta. Por otra parte, otros factores pueden explicar la eficiencia. Por ejemplo, la experiencia y el nivel de conocimientos específicos respecto al nuevo proceso de los operadores pudo dar lugar a mecanismos de regulación colectiva de la eficiencia en los equipos. Nuestras tentativas para examinar el efecto de estos factores se vieron limitados por el tamaño de la muestra. Valdría la pena investigar este punto con una muestra más grande.

\subsection{Contribución teórica}

El aporte principal teórico de la tesis consiste en la elaboración del modelo ARGUinDSM que conceptualiza la actividad argumentativa en situación dinámica. Propuesto desde su aplicación, estos trabajos de tesis han demostrado que se puede operacionalizar en diálogos reales en situaciones ecológicas de conducta de un sistema complejo de alto riesgo. El modelo ARGUinDSM ofrece nuevas perspectivas para comprender los procesos de decisión en sala de control y para mejorar la fiabilización de estos procesos. Por una parte, su aplicación en la operación de una planta nuclear sobrepasa el marco de las situaciones accidentales. De hecho, los operadores también resuelven problemas en operación normal. A veces estos problemas pueden ser más espinosos, en la medida en que las prescripciones operativas son menos estrictas y detalladas en estas situaciones. Por otro lado, el modelo ARGUinDSM se puede generalizar a otros entornos dinámicos en los cuales los lapsos de tiempo son suficientemente amplios para que los operadores puedan ejercer una actividad de resolución de problemas colectiva.

\section{BIBLIOGRAFÍA}

Baker, M. J. (1999). Argumentation and constructive interaction. In P. Coirier \& J. Andriessen (Eds.), Foundations of Argumentative Text Processing (pp. 179-202). Amsterdam: University of Amsterdam Press.

Barth, S., Schraagen, J. M., \& Schmettow, M. (2015). Network measures for characterising team adaptation processes. Ergonomics, 58(8), 1287-1302. https://doi.org/

10.1080/00140139.2015.1009951

Bourgeon, L., Valot, C., \& Navarro, C. (2013). Communication and Flexibility in Aircrews Facing Unexpected and Risky Situations. The International Journal of Aviation Psychology, 23(4), 289-305. https://doi.org/10.1080/10508414.2013.833744

Breton, P. (2006). L'argumentation dans la communication. Paris, France : Editions La Découverte. 
Cannon-Bowers, J. A., Salas, E., \& Converse, S. (1993). Shared mental models in expert team decision making. In N. J. Castellan (Ed.), Individual and group decision making: Current issues. Hillsdale, N: Erlbaum.

Champaud, C. (1994). L'argumentation. Psychologie Française, 39(2), 193-203.

Darses, F. (2006). Analyse du processus d'argumentation dans une situation de reconception collective d'outillages. Le travail hu main, 69(4), 317-347. https://doi.org/10.3917/th.694.0317

De La Garza, C., Labarthe, J.-P., \& Graglia, L. (2012). The contribution of ergonomics to risk analysis in the design process: the case of a future control room. Work: A Journal of Prevention, Assessment and Rehabilitation, 41 Suppl 1, 730-736. https://doi.org/10.3233/WOR-2012-0233-730

Hagler, D. A. \& Brem, S. K. (2008). Reaching agreement: The structure \& pragmatics of critical care nurses' informal argument. Contemporary Educational Psychology, 33(3), 403-424. https:// doi.org/10.1016/j.cedpsych.2008.05.002

Hoc, J.-M. \& Amalberti, R. (1995). Diagnosis: Some theoretical questions raised by applied research. Cahiers de Psychologie Cognitive, 14(1), 73-101.

Klein, G. \& Klinger, D. (1991). Naturalistic Decision Making. Human Systems Information Analysis Center Gateway, XI(3), 16-19.

Labarthe, J.-P. \& De La Garza, C. (2011). The human factors evaluation program of a control room: The French EPR approach. Human Factors and Ergonomics in Manufacturing \& Service Industries, 21(4), 331-349. https://doi.org/10.1002/hfm.20227

Lu, J. \& Lajoie, S. P. (2008). Supporting medical decision making with argumentation tools. Contemporary Educational Psychology, 33(3), 425-442. http://dx.doi.org/10.1016/j.cedpsych. 2008.05.005

Mérand, J. \& Darses, F. (2017). ARGUinDSM - A model of argumentation in team problem-solving situation: An application to nuclear control room teams. Presented at the 13th International Conference on Naturalistic Decision Making, Bath, UK.

Nussbaum, E. M. (2008). Collaborative discourse, argumentation, and learning: Preface and literature review. Contemp rary Educational Psychology, 33(3), 345-359. DOI: 10.1016/j.cedpsych. 2008.06.001

Oléron, P. (1996). L'argumentation. (4e éd). Paris, France : Presses universitaires de France.

Park, J., Jung, W., \& Yang, J.-E. (2012). Investigating the effect of communication characteristics on crew performance under the simulated emergency condition of nuclear power plants. Reliability Engineering \& System Safety, 101, 1-13. https://doi.org/10.1016/j.ress.2012.01.003 Perelman, C. \& Olbrechts-Tyteca, L. (1958/2008). Traité de l'argumentation. La nouvelle rhétorique. 6ème édition. (6e éd). Bruxelles, Belgique : Editions de l'Université de Bruxelles.

Rasmussen, J. (1986). Information Processing and Human-Machine Interaction: An Approach to Cognitive Engineering. New York, NY, USA: Elsevier Science Inc.

Rieke, R. D., Sillars, M. O., \& Peterson, T. R. (2005). Argumentation and critical decision making. (6e éd). Pearson.

Toulmin, S. E. (1958/2003). The uses of argument (2e éd.). New York, USA: Cambridge University Press. https://doi.org/10.1017/CB09780511840005 
Vicente, K. J., Mumaw, R. J., \& Roth, E. M. (2004). Operator monitoring in a complex dynamic work environment: a qualitative cognitive model based on field observations. Theoretical Issues in Ergonomics Science, 5(5), 359-384. https://doi.org/10.1080/14039220412331298929

\author{
AUTOR \\ JOHANNA MÉRAND \\ Institut de Recherche Biomédicale des Armées \\ 91223 Brétigny sur Orge France \\ merand.johanna@gmail.com
}

